\title{
Management Strategies and Outcomes of Hemorrhagic Traumatic Brain Injury on Oral Anticoagulants
}

\author{
Evan M. Krueger ${ }^{1}$, Megan M. Finneran ${ }^{1}$, Michelle Smith ${ }^{2}$ \\ 1. Department of Neurosurgery, Carle Foundation Hospital, Normal, USA 2. Department of Trauma, OSF Healthcare, \\ Bloomington, USA
}

Corresponding author: Evan M. Krueger, dr.evankrueger@gmail.com

\begin{abstract}
Introduction: Traumatic brain injury (TBI) is common, and the frequency of patients taking oral anticoagulants is increasing. However the optimal initial triage, management, and long term care plans of hemorrhagic TBI patients taking oral anticoagulants is not clear.
\end{abstract}

Objectives: To determine the usage pattern of reversal agents for hemorrhagic TBI patients taking oral anticoagulants, and examine their characteristics and outcomes as compared to hemorrhagic TBI patients not taking these medications.

Methods: This was a single-center, retrospective, observational study. Included were adults with trauma categorization and traumatic intracranial hemorrhage (ICH) between April 1, 2017 and December 31, 2019. Patient age, type of ICH, initial Glasgow Coma Scale (GCS) score, oral anticoagulant prescribed pre-injury, anticoagulation reversal agent given, and hospital discharge disposition were recorded.

Results: For the entire sample size ( $\mathrm{n}=111)$, the mean age and GCS were 71.6 years old and 13.8, respectively. Compared to patients not taking oral anticoagulants, patients taking oral anticoagulants were older (76.7 years old versus $69.1 ; p<0.01$ ), had similar GCS scores (13.7 versus $13.9 ; p=0.69)$, had fewer subarachnoid hemorrhages ( $18.9 \%$ versus $37.8 \%$; $p=0.04$ ), were less likely to discharge home ( $48.6 \%$ versus $73.0 \%$; $p=0.01$ ), and had similar incidence of mortality (13.5\% versus 6.7\%; $p=0.30)$. A total of $14 / 37$ (37.8\%) patients taking oral anticoagulants received reversal agents in the emergency department. Compared to patients taking oral anticoagulants and not given reversal agents, patients taking oral anticoagulants and given reversal agents had similar ages ( 78.8 years old versus $75.4 ; p=0.41$ ), had similar GCS scores (12.9 versus $14.1 ; p=0.17$ ), had similar ICH types (all $p=1.0$ ), were less likely to discharge home ( $48.6 \%$ versus $73.0 \% ; p=0.01$ ), and had higher incidence of mortality ( $28.6 \%$ versus $4.2 \% ; p=0.05)$.

Conclusions: This limited data set did not show improved outcomes by giving reversal agents to hemorrhagic TBI patients taking oral anticoagulants. However, until more robust data is available, judicious use of reversal agents in this high-risk patient population should remain common practice.

Received 08/26/2020

Review began 08/31/2020

Review ended 09/03/2020

Published 09/17/2020

(c) Copyright 2020

Krueger et al. This is an open access

article distributed under the terms of the Creative Commons Attribution License CC-BY 4.0., which permits unrestricted use, distribution, and reproduction in any medium, provided the original author and source are credited.
Categories: Neurosurgery, Trauma

Keywords: traumatic brain injury, anticoagulation, antiplatelet, intracranial hemorrhage

\section{Introduction}

The yearly incidence of traumatic brain injury (TBI) in the United States is 1.6 million, resulting in 290,000 hospitalizations [1]. In 2010, the yearly economic burden in the United States for non-fatal TBI was nearly $\$ 88$ billion [2]. As the general population ages, more patients are being prescribed oral anticoagulants [3]. However, management strategies for anticoagulant induced side-effects, such as intracranial hemorrhage (ICH), are not fully validated. There is even further paucity of data on the appropriate triage, management, and long term care for hemorrhagic TBI patients taking oral anticoagulants.

The purpose of this study was to share a single-center experience with managing hemorrhagic TBI patients on oral anticoagulants. We sought to determine the usage pattern of reversal agents for hemorrhagic TBI patients taking oral anticoagulants, and examine their characteristics and outcomes as compared to hemorrhagic TBI patients not taking these medications. The aim of this study is to improve care and resource utilization in hemorrhagic TBI patients.

\section{Materials And Methods}

This was a single-center, retrospective, observational study of a prospectively maintained outcomes database that obtained IRB approval (\#0000068). All consecutive patients from the healthcare system trauma registry at a Level II trauma center between April 1, 2017 and December 31, 2019 were queried. Inclusion 
criteria were: age $\geqslant 18$, categorized trauma by the emergency department (ED), and the presence of any type of acute traumatic ICH. Exclusion criteria were: presence of pre-existing intracranial blood or mass lesion, and incomplete records. The study variables recorded were patient age, type of ICH, initial Glasgow Coma Scale (GCS) score, oral anticoagulant prescribed pre-injury, anticoagulation reversal agent given in the ED (including human blood products and synthetic agents), and patient hospital discharge disposition. Patient groups were categorized as taking oral anticoagulants (OAC), not taking oral anticoagulants (n-OAC), taking oral anticoagulants and given reversal agents (OAC-r), or taking oral anticoagulants and not given reversal agents (OAC-nr).

A sample of convenience was utilized. An a priori power analysis was not performed since consecutive patients were reviewed. To compare mean age and GCS, a student's t-test was used. A Fischer's exact test or chi-square test were used to compare type of ICH and hospital discharge disposition. All calculations were performed using SAS (version 9.4; SAS Institute Inc., Cary, NC, US). Means (range, \pm 1 standard deviation) are reported. $P \leqslant 0.05$ was considered statistically significant.

\section{Results}

A total of 111 patients met inclusion criteria. The mean age and GCS were 71.6 (23-95 years old, \pm 14.1$)$ and 13.8 (3-15, \pm 2.5 ), respectively. There were 77/111 (69.4\%) subdural hematomas (SDH), 35/111 (31.5\%) traumatic subarachnoid hemorrhages (SAH), and 8/111 (13.8\%) intraparenchymal hemorrhages (IPH). For hospital discharge disposition, 72/111 (64.9\%) went home, 23/111 (20.1\%) went to a skilled nursing facility (SNF), 4/111 (3.6\%) went to in-patient rehab, and 2/111 (1.8\%) were transferred to another facility directly from the ED. There were 10/111 (9.0\%) mortalities, with a mean GCS score of 9.

Thirty-seven of 111 (33.3\%) patients were taking oral anticoagulants prior to injury. The distribution of OAC were as follows: 17/37 (46.0\%) clopidogrel, 9/37 (24.3\%) warfarin, 8/37 (21.6\%) apixiban, 2/37 (5.4\%) dabigatran, $2 / 37$ (5.4\%) rivaroxaban, $1 / 37$ (2.7\%) aspirin-dipyridamole, and 1/37 (2.7\%) ticagrelor. OAC were older than n-OAC (76.7 years old, 38-95, \pm 11.8 ; versus $69.1,23-90, \pm 14.5 ; p<0.01)$. OAC had similar GCS scores compared to n-OAC $(13.7,6-15, \pm 2.6$; versus $13.9,3-15, \pm 2.5 ; p=0.69)$. As compared to $\mathrm{n}$-OAC, OAC had similar rates of $\operatorname{SDH}(27 / 37,73.0 \%$; versus 50/74, 67.6\%; $p=0.47)$, had fewer SAH (7/37, 18.9\%; versus $28 / 74,37.8 \%$; $p=0.04)$, and had similar rates of IPH $(4 / 37,10.8 \%$; versus $4 / 74,5.4 \% ; p=0.44)$. As compared to n-OAC, OAC were less likely to discharge home (18/37, 48.6\%; versus $54 / 74,73.0 \% ; p=0.01)$, were more likely to discharge to SNF or rehab (14/37, 37.8\%; versus $13 / 74,17.5 \%$; $p<0.01)$, and had similar incidence of mortality $(5 / 37,13.5 \%$; versus $5 / 74,6.7 \% ; p=0.30)$.

A total of 14/37 (37.8\%) patients taking oral anticoagulants received reversal agents in the ED (Table 1). 


\section{Cureus}

\begin{tabular}{|c|c|c|c|c|c|}
\hline Age & Hemorrhage & GCS & OAC & Reversal Agent & Disposition \\
\hline 75 & SAH & 9 & apixiban & PCC & mortality \\
\hline 80 & SDH & 6 & apixiban & PCC & mortality \\
\hline 86 & SDH & 14 & apixiban & PCC & SNF \\
\hline 61 & $\mathrm{IPH}$ & 15 & clopidogrel & platelets & rehab \\
\hline 84 & SDH & 14 & clopidogrel & platelets & SNF \\
\hline 86 & SDH & 15 & clopidogrel & platelets & SNF \\
\hline 81 & SDH & 13 & clopidogrel, warfarin & $F F P$, vit $K$ & SNF \\
\hline 81 & SAH & 15 & dabigatran & idarucizumab & home \\
\hline 88 & SDH & 14 & rivaroxaban & platelets & SNF \\
\hline 85 & SAH & 15 & warfarin & FFP & home \\
\hline 65 & SDH & 15 & warfarin & vit K & home \\
\hline 74 & SDH & 15 & warfarin & vit K, PCC & home \\
\hline 78 & SDH & 6 & warfarin & FFP & mortality \\
\hline 79 & SDH & 15 & warfarin & FFP, vit K, pRBC & mortality \\
\hline
\end{tabular}

\section{TABLE 1: Hemorrhagic Traumatic Brain Injury Patients on Oral Anticoagulation Medicine Given}

\section{Reversal Agents.}

Abbreviations: IPH, intraparenchymal hemorrhage; FFP, fresh frozen plasma; GCS, glasgow coma scale; OAC, oral anticoagulant; PCC, prothrombin complex concentrate; pRBC, packed red blood cell; SAH, subarachnoid hemorrhage; SDH, subdural hematoma; SNF, skilled nursing facility; vit K, vitamin $\mathrm{K}$

Specifically, 6/9 (66.6\%) patients taking vitamin-k antagonists, 4/10 (40\%) patients taking factor X inhibitors, $4 / 19(21.1 \%)$ patients taking antiplatelet medications, and 1/2 (50\%) patients taking direct thrombin inhibitors pre-morbid were reversed. The OAC-nr group is summarized in Table 2. 


\section{Cureus}

\begin{tabular}{|c|c|c|c|c|}
\hline Age & Hemorrhage & GCS & OAC & Disposition \\
\hline 64 & SDH & 14 & apixaban & home \\
\hline 63 & SDH & 15 & apixaban & home \\
\hline 90 & SDH & 15 & apixaban & home \\
\hline 87 & SDH & 15 & apixaban & home \\
\hline 86 & SDH & 15 & apixaban & SNF \\
\hline 69 & SDH & 15 & aspirin, dipyridamole & home \\
\hline 38 & $\mathrm{IPH}$ & 15 & clopidogrel & home \\
\hline 61 & SAH & 15 & clopidogrel & home \\
\hline 84 & SDH & 14 & clopidogrel & home \\
\hline 73 & SDH & 15 & clopidogrel & home \\
\hline 83 & SDH & 15 & clopidogrel & home \\
\hline 78 & SDH & 15 & clopidogrel & mortality \\
\hline 72 & $\mathrm{IPH}$ & 12 & clopidogrel & SNF \\
\hline 77 & SDH & 11 & clopidogrel & SNF \\
\hline 76 & SDH & 12 & clopidogrel & SNF \\
\hline 93 & SDH & 15 & clopidogrel & SNF \\
\hline 86 & SDH, SAH & 15 & clopidogrel & SNF \\
\hline 71 & SDH & 15 & clopidogrel, rivaroxaban & home \\
\hline 88 & SDH & 15 & clopidogrel, warfarin & home \\
\hline 95 & SDH & 15 & dabigatran & home \\
\hline 78 & SAH & 15 & ticagrelor & SNF \\
\hline 71 & SAH & 15 & warfarin & home \\
\hline 52 & $\mathrm{IPH}$ & 7 & warfarin & SNF \\
\hline
\end{tabular}

TABLE 2: Hemorrhagic Traumatic Brain Injury Patients on Oral Anticoagulation Medicine not Given Reversal Agents.

Abbreviations: IPH, intraparenchymal hemorrhage; GCS, glasgow coma scale; OAC, oral anticoagulant; SAH, subarachnoid hemorrhage; SDH, subdural hematoma; SNF, skilled nursing facility.

OAC-r had similar ages compared to OAC-nr (78.8 years old, 61-88, \pm 7.9 ; versus $75.4,38-95, \pm 13.7 ; p=0.41$ ). OAC-r had similar GCS scores compared to OAC-nr $(12.9,6-15, \pm 3.3$; versus $14.1,7-15, \pm 1.9 ; p=0.17)$. Compared to OAC-nr, OAC-r had similar rates of SDH (10/14, 71.4\%; versus $17 / 23,70.8 \%$; $p=1.0)$, SAH (3/14, $21.4 \%$; versus $4 / 23,16.7 \% ; p=1.0$ ), and IPH (1/14, 7.1\%; versus $3 / 23,12.5 \% ; p=1.0)$. Compared to OAC-nr, OAC-r were less likely to discharge home $(4 / 14,28.6 \%$; versus $14 / 23,60.9 \%, p=0.05)$, had similar rates of discharge to SNF or rehab $(6 / 14,42.8 \%$; versus $8 / 23,34.8 \% ; p=0.38)$, and had higher incidence of mortality (4/14, 28.6\%; versus $1 / 23,4.2 \%$; $p=0.05)$.

\section{Discussion}

During initial triage of hemorrhagic TBI patients, early clinical decision making is often inferred from limited information. While outcomes of all TBI vary tremendously, mild TBI - defined as GCS 13-15 - have reported only 3.5\% rates of neurosurgical intervention [4]. Furthermore, hemorrhagic mild TBI patients have reported only $1.5 \%$ rates of unexpected delayed neurosurgical intervention [5]. In our study, OAC and n-OAC patients surprisingly had similar GCS scores. Newer oral anticoagulants such as apixaban, dabigatran, or rivaroxaban have previously been reported to not have higher rates of traumatic ICH occurrence, progression, or death as compared to aspirin, clopidogrel, and warfarin [6]. Additionally, pre-injury 
antiplatelet usage has been shown to be associated with increased mortality [7]. Congruently, in our study, OAC patients were less likely to be discharged home as compared to n-OAC patients.

Whether to give reversal agents to hemorrhagic TBI patients is complex, and is based upon many factors including immediate and foreseeable risk of clinical and radiographic deterioration, and need for neurosurgical intervention. A clinician weighs these against reversal agent complications such as thromboembolism, financial cost, and potential limited availability and efficacy. In our study, there were no differences in age, GCS, and ICH types for OAC-r and OAC-nr. Despite their similarities, OAC-r had worse hospital discharge dispositions and higher incidence of mortality compared to OAC-nr. Our data however is limited, and any conclusions drawn exclusively from it should be viewed as equivocal. In a retrospective study, it was noted that while patients receiving platelet transfusions for pre-morbid aspirin or clopidogrel use had higher injury severity scores and lower GCS, their mortality was significantly higher as compared to patients not receiving platelet transfusions [7]. Platelet transfusion does not result in decreased expansion of non-operative traumatic SDH [8]. Conversely, others have reported improved outcomes and reduced mortality with platelet transfusions for hemorrhagic TBI patients on P2Y12 inhibitors [9]. For patients older than 60 years old with traumatic ICH, mortality is higher in patients taking vitamin-k antagonists compared to other types of anticoagulants, despite that they more commonly receive reversal agents [10]. As a potential alternative to traditional reversal agents, in an international multicenter trial of TBI patients with a GCS $\leqslant 12$ or any acute ICH, patients were randomized to receive one gram of tranexamic acid (TXA) load within three hours of injury followed by one gram TXA over eight hours versus placebo control. Those receiving TXA had a significant reduction in mortality compared to placebo (12.5\% versus $14.0 \%$, risk ratio 0.89), with a similar risk of vascular occlusive events [11]. Zero patients in our study received TXA. Ultimately despite the limited contrarian data, administration of reversal agents for hemorrhagic TBI patients should, at this time, remain common practice.

In most instances, immediately halting pre-injury oral anticoagulation after hemorrhagic TBI is indicated. However, the timing and method of reinitiating of oral anticoagulants remain unclear. Resuming antiplatelet therapy after traumatic ICH has reported re-hemorrhage risks of $<1 \%$ if within 48 hours, and cumulative $4.7 \%$ if within two weeks [12]. Others have proposed reinitiating oral anticoagulants 9.5 days after injury as an appropriate balance of hemorrhagic and thromboembolic complications [13]. We did not explore timing of resumption of oral anticoagulants in our study.

Managing patients on oral anticoagulants with spontaneous ICH, by comparison, has more evidence available to support clinical decision making. The risk of recurrent spontaneous ICH appears to be centered on individualized risk factors including location of hemorrhage, patient's age, cerebrovascular disease, need for continued anticoagulation, and genetics $[14,15]$. An international multicenter trial of non-surgical patients on antiplatelet therapy with acute spontaneous primary $\mathrm{ICH}$, randomized patients to receive platelet transfusion plus standard medical therapy versus standard medical therapy alone. Patients receiving platelet transfusion had higher odds of death or dependence at three months (odds ratio 2.05, $p=0.01$ ) and a trend towards higher adverse events during their hospital stay [16]. Antiplatelet monotherapy can be reinitiated days after any spontaneous $\mathrm{ICH}$, while the optimal resumption of other oral anticoagulants after non-lobar ICH has been suggested to range from 72 hours to four weeks [14,15]. Warfarin should not be continued for the treatment of nonvalvular atrial fibrillation after a warfarin-induced lobar ICH [14].

This study shared the experience of managing hemorrhagic TBI patients at a Level II trauma center. In the United States, there is a growing trend of regionalization of trauma hospitals despite that the majority of neurosurgeons do not practice at Level I trauma centers [17]. Most neurosurgeons may not have a robust blood bank in their armamentarium. Additionally, financial cost can unfortunately cloud clinical decision making. In 2020, the costs per patient to give prothrombin concentrate and andexanet-alpha were $\$ 5,670$ and $\$ 22,129$, respectively; the later exceeded Medicare total hospital reimbursement in $74 \%$ of patients by $\$ 7,604$ [18]. There may be a select group of hemorrhagic TBI patients on oral anticoagulants whose risk of neurologic decline is less than the risk associated with administration of a reversal agent. Key areas of future focus should include improving resource utilization of scare, expensive, and potentially dangerous oral anticoagulant reversal agents.

There are several limitations to this study. Foremost, this was a retrospective observational study with a relatively small sample size, which carries inherent limitations. Next, our database did not record potential confounders including, (1) pupillary response, which may have resulted in very poor prognosis patients (e.g., GCS 3 or 4 with fixed unreactive pupils) being included for analysis, and (2) serum ethanol levels or other pre-existing medical comorbidities such as hematologic conditions or liver cirrhosis, that may have affected a patient's coagulation profile and risk of hemorrhage, (3) the size of the traumatic ICH, and (4) the mechanism or severity of traumatic injury. Third, we lumped all oral anticoagulants and reversal agents together for statistical analysis, despite their significant heterogeneity. Another limitation is that we did not consider the clinicians' justifications for giving a reversal agent, such as if it was based on clinical exam, laboratory values, or some combination. Fifth, we did not have a way to record where the patient was living prior to admission, and therefore a patient who was discharged to a skilled nursing facility may have actually returned to their pre-injury home. Lastly, our database did not record the patient's clinical or functional status at discharge. 


\section{Conclusions}

In conclusion, we present a retrospective, single-center review of reversal strategies and short-term outcomes of TBI patients on oral anticoagulants. In a relatively small sample size, we showed OAC patients were older than n-OAC patients and tended to have worse hospital discharge dispositions. The decision to administer reversal agents did not appear to be dictated by age, GCS, or type of ICH. Receiving reversal agents was not associated with improved discharge disposition or lower mortality. However judicious use of reversal agents in this high-risk patient population should still remain common practice. For the foreseeable future, clinical decision making for hemorrhagic TBI patients on oral anticoagulants will be informed primarily from a collection of lower powered studies.

\section{Additional Information \\ Disclosures}

Human subjects: Consent was obtained by all participants in this study. Animal subjects: All authors have confirmed that this study did not involve animal subjects or tissue. Conflicts of interest: In compliance with the ICMJE uniform disclosure form, all authors declare the following: Payment/services info: All authors have declared that no financial support was received from any organization for the submitted work. Financial relationships: All authors have declared that they have no financial relationships at present or within the previous three years with any organizations that might have an interest in the submitted work. Other relationships: All authors have declared that there are no other relationships or activities that could appear to have influenced the submitted work.

\section{Acknowledgements}

The authors thank Vanessa K. Pazdernik MS, from the Department of Research Support A.T. Still University, who designed and performed statistical analysis.

\section{References}

1. Rutland-Brown W, Langlois JA, Thomas KE, Xi YL: Incidence of traumatic brain injury in the United States, 2003. J Head Trauma Rehabil. 2006, 21:544.

2. Centers for Disease Control and Prevention. Data \& statistics (WISQARS): cost of injury reports . (2010). Accessed: September 20, 2016: https://wisqars.cdc.gov:8443/costT/.

3. Xu Y, Holbrook AM, Simpson CS, Dowlatshahi D, Johnson AP: Prescribing patterns of novel oral anticoagulants following regulatory approval for atrial fibrillation in Ontario, Canada: a population-based descriptive analysis. CMAJ Open. 2013, 1:115-119. 10.9778/cmajo.20130032

4. Marincowitz C, Lecky FE, Townend W, Borakati A, Fabbri A, Sheldon TA: The risk of deterioration in GCS13-15 patients with traumatic brain injury identified by computed tomography imaging: a systematic review and meta-analysis. J Neurotrauma. 2018, 35:703-718. 10.1089/neu.2017.5259

5. Krueger EM, Putty M, Young M, Gaynor B, Omi E, Farhat H: Neurosurgical outcomes of isolated hemorrhagic mild traumatic brain injury. Cureus. 2019, 11:5982. 10.7759/cureus.5982

6. Kobayashi L, Barmparas G, Bosarge P, et al.: Novel oral anticoagulants and trauma: the results of a prospective American Association for the Surgery of Trauma Multi-Institutional trial. J Trauma Acute Care Surg. 2017, 82:827-835. 10.1097/ta.0000000000001414

7. Fortuna GR, Mueller EW, James LE, Shutter LA, Butler KL: The impact of preinjury antiplatelet and anticoagulant pharmacotherapy on outcomes in elderly patients with hemorrhagic brain injury. Surgery. 2008, 144:598-603. 10.1016/j.surg.2008.06.009

8. Ogunlade J, Wiginton JG, Ghanchi H, Al-Atrache Z, Wacker M, Menoni R, Miulli D: Efficacy of platelet transfusion in the management of acute subdural hematoma. Clin Neurol Neurosurg. 2018, 174:163-166. 10.1016/j.clineuro.2018.09.021

9. Jehan F, Zeeshan M, Kulvatunyou N, et al.: Is there a need for platelet transfusion after traumatic brain injury in patients on P2Y12 inhibitors?. J Surg Res. 2019, 236:224-229. 10.1016/j.jss.2018.11.050

10. Prexl O, Bruckbauer M, Voelckel W, Grottke O, Ponschab M, Maegele M, Schochl H: The impact of direct oral anticoagulants in traumatic brain injury patients greater than 60-years-old. Scand J Trauma Resusc Emerg Med. 2018, 26:20. 10.1186/s13049-018-0487-0

11. The CRASH-3 Trial Collaborators: Effects of tranexamic acid on death, disability, vascular occlusive events and other morbidities in patients with acute traumatic brain injury (CRASH- 3): a randomised, placebocontrolled trial. Lancet. 2019, 394:1713-1723. 10.1016/s0140-6736(19)32233-0

12. Kerr K, Wilkerson C, Shepard S, Choi H, Kitagawa R: Use of anti-platelet agents after traumatic intracranial hemorrhage. Clin Neurol Neurosurg. 2016, 140:85-90. 10.1016/j.clineuro.2015.11.012

13. Puckett Y, Zhang K, Blasingame J, et al.: Safest time to resume oral anticoagulation in patients with traumatic brain injury. Cureus. 2018, 10:2920. 10.7759/cureus.2920

14. Hemphill JC, Greenberg SM, Anderson CS, et al.: Guidelines for the management of spontaneous intracerebral hemorrhage: a guideline for healthcare professionals from the American Heart Association/American Stroke Association. Stroke. 2015, 46:2032-2060. 10.1161/str.0000000000000069

15. Hawryluk GW, Austin JW, Furlan JC, Lee JB, O'Kelly C, Fehlings MG: Management of anticoagulation following central nervous system hemorrhage in patients with high thromboembolic risk. J Thromb Haemost. 2010, 8:1500-1508. 10.1111/j.1538-7836.2010.03882.x

16. Baharoglu MI, Cordonnier C, Al-Shahi Salman R, et al.: Platelet transfusion versus standard care after acute stroke due to spontaneous cerebral haemorrhage associated with antiplatelet therapy (PATCH): a randomised, open-label, phase 3 trial. Lancet. 2016, 387:2605-2613. 10.1016/s0140-6736(16)30392-0 


\section{Cureus}

17. Babu MA, Stroink AR, Timmons SD, Orrico KO, Prall JA: Neurosurgical coverage for emergency and trauma call. Neurosurgery. 2019, 84:977-984. 10.1093/neuros/nyy354

18. Frontera JA, Bhatt P, Lalchan R, Yaghi S, Ahuja T, Papadopoulos J, Joset D: Cost comparison of andexanet versus prothrombin complex concentrates for direct factor Xa inhibitor reversal after hemorrhage. J Thromb Thrombolysis. 2020, 49:121-131. 10.1007/s11239-019-01973-z 